\title{
From Photoemission Microscopy to an "All-in-One" Photoemission Experiment
}

\author{
Christian Tusche, ${ }^{\mathrm{a}, \mathrm{b}}$ Ying-Jiun Chen, ${ }^{\mathrm{a}, \mathrm{b}}$ Lukasz Plucinski, ${ }^{\mathrm{a}, \mathrm{b}}$ Claus M. Schneider ${ }^{\mathrm{a}, \mathrm{b}, \mathrm{c}, \dagger}$ \\ a Peter Grünberg Institute, Research Center Jülich, D-52425 Jülich, Germany \\ ${ }^{\mathrm{b}}$ Faculty of Physics, University of Duisburg, D-47057 Duisburg, Germany \\ c Physics Department, University of California, Davis, USA \\ ${ }^{\dagger}$ Corresponding author: c.m.schneider@fz-juelich.de
}

Received: 11 January, 2020, Accepted 30 January, 2020, Published 29 February, 2020

Photoelectron spectroscopy is our main tool to explore the electronic structure of novel material systems, the properties of which are often determined by an intricate interplay of competing interactions. Elucidating the role of this interactions requires studies over an extensive range of energy, momentum, length, and time scales. We show that immersion lens-based momentum microscopy with spin-resolution is able to combine these seemingly divergent requirements in a unifying experimental approach. We will discuss applications to different areas in information research, for example, resistive switching and spintronics. The analysis of resistive switching phenomena in oxides requires high lateral resolution and chemical selectivity, as the processes involve local redox processes and oxygen vacancy migration. In spintronics topological phenomena are currently a hot topic, which lead to complex band structures and spin textures in reciprocal space. Spin-resolved momentum microscopy is uniquely suited to address these aspects.

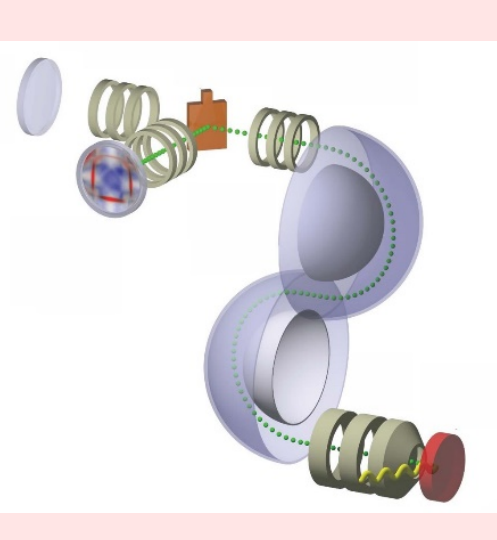

Keywords Angle-resolved photoemission; Momentum microscopy; Spin polarization analysis; Spin-orbit coupling, Magnetism

\section{INTRODUCTION}

The physical properties of modern condensed matter systems are largely determined by the details of their electronic structure. This is true for both the ground and excited state. Phenomena such as magnetism or superconductivity are the result of a complex interplay of chargeand spin-dependent interactions in a many-electron system on different length scales and even time scales, if dynamic processes are considered. The overarching concepts of symmetry and topology govern the novel field of quantum materials and may result in complex spin behaviors in real and momentum space [1].

By now, we know a large suite of photon-in/electron-out techniques, which probe different aspects of the electronic structure and their connection to specific physical and chemical properties. In the past, two main methodological avenues have been followed rather independently. Photoelectron spectroscopy is the method of choice if it comes to a detailed characterization of the electronic states in quantum materials [2]. Recent years have seen amazing developments in the technique of angle-resolved photoelectron spectroscopy (ARPES) with respect to both spectrometers and light sources. With the help of narrow bandwidth lasers the energy resolution limit has been pushed to less than $1 \mathrm{meV}$ [3]. Using focused high-brilliance synchrotron radiation, a lateral resolution in the $100 \mathrm{~nm}$ range could be obtained in scanning ARPES (nanoARPES) systems $[4,5]$. A lateral mapping in momentum $(k)$ space became available by the recent introduction of scanning lens options.

Understanding electronic states with respect to all quantum numbers requires the electron spin to be explicitly taken in to account. The use of spin-resolved ARPES was for a long time limited by the low efficiency of the various types of spin detection schemes which involved spin-orbit scattering [6]. A first improvement in single-channel spindetection came finally with the low-energy iron detector based on exchange scattering [7]. We successfully implemented single-channel spin detectors into hemispherical spectrometer systems [8], including the most efficient version of the iron detector [9]. This system was recently 


\section{eJSSNT}

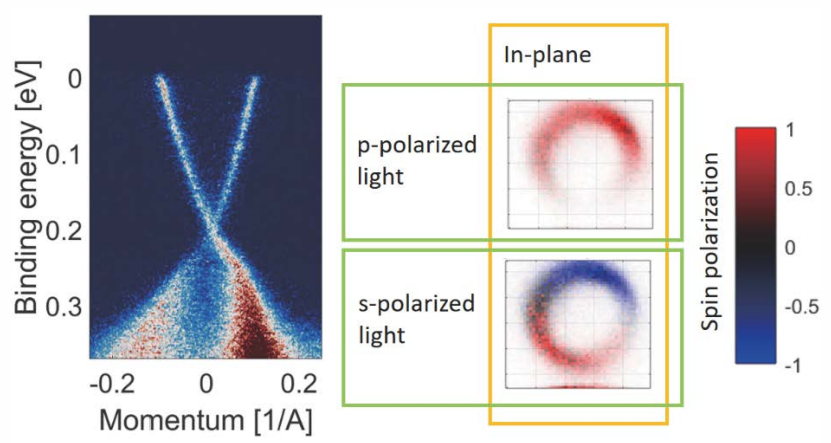

Figure 1: High-resolution spin-resolved photoemission from the (0001) surface of the topological insulator $\mathrm{Bi}_{2} \mathrm{Se}_{3}$. Left: Dispersion of the topological surface state (TSS) close to the Fermi level. Right: Spin-polarization maps of the TSS at $E_{\mathrm{F}}$ in the $\left(k_{x}, k_{y}\right)$ plane.

upgraded by a scanning lens option and a $6 \mathrm{eV} \mathrm{cw}$ laser providing the means for high-resolution spin-polarization studies. An example is given Figure 1 showing the topological surface state in $\mathrm{Bi}_{2} \mathrm{Se}_{3}(0001)$ [10]. The ring-like Fermi surface of this Dirac cone in the $\left(k_{x}, k_{y}\right)$ plane is mapped by scanning the electron trajectories in the entrance lens of the spectrometer and displays the characteristic spin texture due to spin-momentum locking. The difference in the spin data for s- and p-polarized light is caused by the linear dichroism in the photoelectron angular distribution related to the angle of light incidence [11].

A second independent development concerned photoemission microscopy (PEEM) and aimed at imaging surfaces with high spatial resolution. This was achieved by the immersion lens concept originally developed by Brüche [12] Whereas early PEEM instruments did not discriminate the photoelectron energies, more advanced concepts implemented energy-dispersive elements and added spectroscopic capabilities in the PEEM [13]. However, even without additional energy filters, using PEEM with synchrotron radiation in an X-ray absorption spectroscopy mode resulted in a very powerful methodology with widespread use in many scientific disciplines. An example from materials science for information technology is given in Figure 2 and studies the electronic changes related to resistive switching in oxides - in this case specifically the perovskite LSMO $\left(\mathrm{La}_{0.7} \mathrm{Sr}_{0.3} \mathrm{MnO}_{3}\right)$ [14]. Resistive switching denotes reversible nonvolatile changes of the electrical conductivity of a material controlled by voltage or current pulses [15]. In oxides this effect is often caused by local redox reactions and the creation/motion of oxygen vacancies. The lateral resolution provided by PEEM enables spectromicroscopy on small sample areas.

The changes between the high resistive (HRS) and low resistive states (LRS) are clearly visible at the Mn L-edge absorption spectra, which have been recorded from small areas, which were subjected to voltage pulses of different height. In particular, the $\mathrm{L}_{3}$ absorption line exhibits a clear fine structure, which reflects a change of the oxidation state from $\mathrm{Mn}^{3+}$ (LRS) to $\mathrm{Mn}^{2+}$ (HRS). Since the $\mathrm{Mn}$ ion is also responsible for the magnetization in LSMO, the resistive switching should also affect the magnetic moments. This can be explored by X-ray magnetic circular dichroism (XMCD), and indeed we find a significant change in the XMCD signature at the $\mathrm{L}_{2,3}$ edges. A quantitative interpretation of these spectral data will need further theoretical input.

\section{MOMENTUM MICROSCOPY}

The immersion lens column of a PEEM generates an image of the lateral distribution of the emitted photoelectrons $I(x, y)$. By adding an additional lens, however, the spatial image can be Fourier transformed into an image of the photoelectron emission angles. As the emission angle is directly related to the photoelectron momentum value in the surface plane $k_{\|}=\left(k_{x}, k_{y}\right)$, the resulting image contains the momentum distribution of the photoelectrons with high angular resolution [16]. In "momentum microscopy" this image is energy-filtered to yield an energy- and momentum-resolved two-dimensional (2D) photoelectron intensity distribution $I\left(E_{\mathrm{kin}}, k_{x}, k_{y}\right)$ for a fixed photoelectron kinetic energy. Conventional ARPES can only acquire small segments in reciprocal space even when a scanning lens option is used. The parallel acquisition approach of momentum microscopy enables a highly efficient mapping of entire Brillouin zone volumes $B\left(E_{\mathrm{B}}, k_{x}, k_{y}\right)$ ( $E_{\mathrm{B}}$; binding energy) by scanning the kinetic energy. Currently, with respect to the energy resolution two main development lines of momentum microscopes are followed. The first line is based on conventional electrostatic energy filters and will be further discussed be-

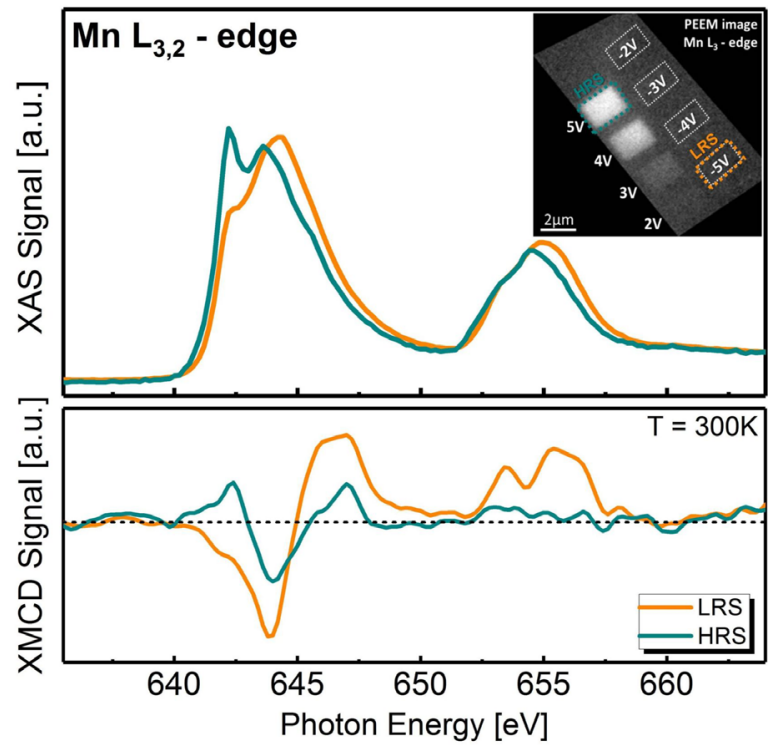

Figure 2: Top: X-ray absorption microspectra at the $\mathrm{Mn} \mathrm{L}_{2,3}$ edges taken on resistively switched areas on LSMO. Inset: PEEM image showing the rectangular areas subjected to different voltage pulses, marking also the high resistive (HRS) and low resistive (LRS) states. Bottom: XMCD microspectra from the HRS and LRS regions, which have been remanently magnetized. 
low. The second line exploits the time-of-flight (ToF) principle for the energy discrimination in combination with a pulsed light source. In this case the photoelectrons generated by the light pulse travel through a drift tube, before they hit a 2D delay line detector (DLD) [17]. Depending on their kinetic energy - or velocity - the electrons reach the DLD at different time $t$, yielding a data set $I\left(t, k_{x}, k_{y}\right)$. The DLD is synchronized with the pulsed light source, which enables one to convert the data set into the energy- and momentum-resolved photoelectron map $I\left(E_{\mathrm{kin}}, k_{x}, k_{y}\right)$. Given a pulsed light source the time-of-flight approach provides the means for a very efficient momentum microscopy at least with respect to intensity measurements, which has been demonstrated recently in Ref. 18.

A spin-resolved version of the momentum microscopy poses a major challenge, as the conventional single-channel spin polarimeters are of very limited use. The necessary breakthrough in spin polarimetry had to wait for the development of 2D spin analysis schemes, which became available only recently $[19,20]$. In this "imaging" approach a 2D quasi-monoenergetic electron distribution $I\left(E_{\mathrm{s}}, k_{x}, k_{y}\right)$ is specularly scattered off the polarimetry target, which results in a spin polarization distribution $P_{n}\left(k_{x}, k_{y}\right)$ ( $n$ : quantization axis along which the spin polarization is determined). Combining an imaging spin detector with a momentum microscope not only marks the long-awaited breakthrough in spin-resolving ARPES experiments, as demonstrated in Ref. 21. In fact, it also paves the way to an "All-in-One" photoemission experiment. With such an instrument, we may be able to investigate spin-dependent electronic states and processes in real space $(\vec{r})$ and reciprocal space $(\vec{k})$ as a function of binding energy $E$. Using pulsed light sources and appropriate pump-probe schemes we may also add the parameter time $t$, eventually to time scales in the sub-picosecond regime. This unique combination will provide a completely new tool set to investigate and disentangle the complex interplay of spin-dependent electronic interactions.

\section{EXPERIMENTAL REALIZATION}

A spin-resolved momentum microscope comprises three principal elements: (i) immersion lens optics, (ii) energy filter, and (iii) imaging spin detector (Figure 3). The immersion lens optics is very similar to the columns used in photoemission microscopy (PEEM) [13]. It generates a strong electrostatic field (up to $10 \mathrm{kV} \mathrm{mm} \mathrm{mm}^{-1}$ ) between sample surface and lens to collect all the photoelectrons emitted from the sample. For a subsequent spin polarization analysis, a purely electrostatic lens system is advantageous as it precludes spin precession which would otherwise occur in magnetic fields. It is important to note that the immersion lens system preserves both the spatial and angular information of the electrons passing through it. Depending on the setting it can therefore generate a $2 \mathrm{D}$ image of the spatial or angular distribution of the photoelectrons at its output. The first one corresponds to the classical PEEM mode, whereas the second one is the basis for the momentum microscopy mode.

The 2D electron distribution at the output of the immersion lens imaging column is then fed into an energy filter, which in our case is a double-pass hemispherical deflection analyzer (HDA). A transfer lens system between the two HDAs permits us to choose between high energy resolution or high transmission modes, while preserving the $2 \mathrm{D}$ characteristics of the photoelectron distribution [22]. Behind the output of the double-pass HDA a transfer lens system transports the electrons into a set of projection lenses, in order to form a magnified spin-integrated intensity image on a $2 \mathrm{D}$ detector in the straight direction [Figure 3(a)].

For the spin polarization analysis, a scattering target, e.g., a $\mathrm{W}(001)$ or $\operatorname{Ir}(001)$ surface, is placed into the imaging column behind the HDAs. The detection principle involves a scattering geometry of $90^{\circ}$ and requires a well-defined working point, i.e., scattering energy $E_{\mathrm{s}}$. The energy-filtered photoelectrons are quasi-monochromatic and are then accelerated/decelerated to the appropriate scattering energy. After the scattering the spin-filtered image is made visible on a two-dimensional detector. The contrast in this image reflects the spin polarization along the quantization axis defined by the spin polarimeter geometry, i.e., the spin polarization component perpendicular to the scattering plane.

We are currently operating a spin-resolving momentum microscope at the synchrotron radiation source Elettra (Italy) $[4,23]$. The undulator beamline covers the range from 50 $\mathrm{eV}$ to $1200 \mathrm{eV}$ and permits the selection of linearly and circularly polarized light. The imaging spin filter employs scattering from a W(001) surface. A second instrument is currently under construction, improving on the successful design originally developed at the MPI Halle (Germany) [21]. It will employ an $\operatorname{Ir}(001)$ imaging spin detector, which provides a high long-term stability of the spin-polarization analysis. The light source will a dedicated high harmonic generation (HHG) source driven by a pulsed laser system with a high repetition rate of up to $10 \mathrm{MHz}$ and covering photon energies up to $100 \mathrm{eV}$ [24]. This set-up will also in-

(a)
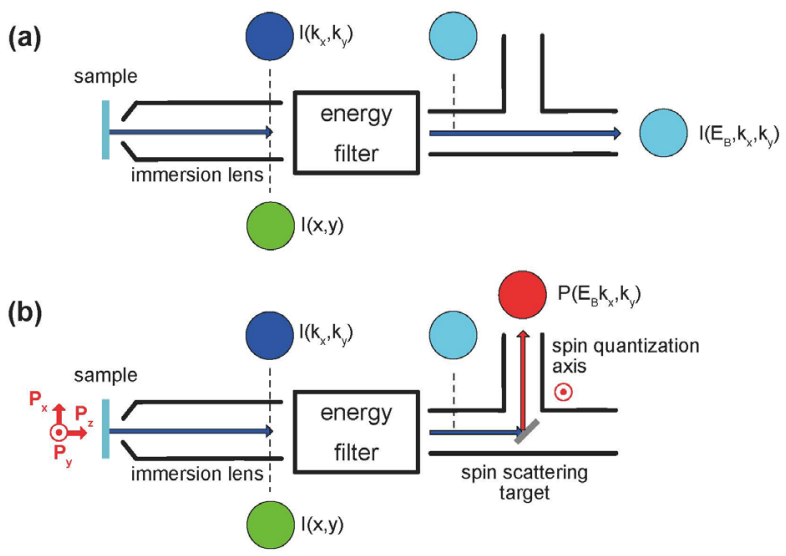

Figure 3: Principal layout of a momentum microscope in the intensity (a) and spin-resolving mode (b), showing the various information channels in real space $I(x, y)$ and reciprocal space $I\left(k_{x}\right.$, $\left.k_{y}\right)$. 


\section{eJSSNT}

clude pump-probe capabilities for carrying out time-resolved studies of electron and spin dynamics.

Given the similar electron-optical concepts for the momentum microscopes involving electrostatic energy filters and the ToF approach, we should also briefly comment on the combination of the imaging spin filter with the ToF scheme. This has already been demonstrated successfully in a study of surface states in W(110) [25]. However, the "polyenergetic" nature of the electrons passing through the drift tube is incompatible with the need for a well-defined scattering energy $E_{\mathrm{s}}$ at the spin-filter target. This situation requires a prefiltering of the electron energy, thereby reducing the efficiency of the original ToF approach for spin-resolved measurements.

\section{APPLICATIONS}

An instrument like the one sketched above enables a multitude of operation modes which brings it close to our desired "All-in-One" spin-resolved photoelectron spectroscopy approach. In the following, we will discuss some of the imaging modes.

\section{A. Real space imaging}

Setting the immersion lens optics to image the lateral distribution of photoelectrons across the sample basically corresponds to the simple PEEM mode. The PEEM mode has been very successfully employed for the imaging of magnetic domains by exploiting the X-ray magnetic circular dichroism (XMCD) in X-ray absorption [26]. This XPEEM approach combines magnetic and element-selective information in a unique manner, mostly probing the unoccupied density of states and measuring the total electron yield without energy filtering. This mode of operation usually provides a large signal and short measuring times. However, polarized synchrotron radiation is required, as the light has to be tuned to characteristic absorption edges in order to obtain a magnetic contrast.

Using the imaging spin detector, such domain imaging experiments can be performed even in the laboratory by means of unpolarized light sources. This has been recently demonstrated on a magnetite sample $\mathrm{Fe}_{3} \mathrm{O}_{4}(001)$ [27]. In contrast to XPEEM, the information stems from well-defined spin-polarized valence electronic states in the band structure of magnetite, which are singled out by means of the energy filter. The surface sensitivity of the photoemission approach reveals a rearrangement of the magnetic surface domains, when passing through the Verwey transition. The spin polarization of the valence electronic states, however, appeared to be the same above and below the Verwey temperature.

We note that the spin-polarized imaging approach may also provide a higher lateral resolution than XPEEM, if a continuous wave laser source is used for illumination. In this case space charge problems can be avoided, which generally limit the lateral resolution in XPEEM due to the pulse nature of the synchrotron light [28]. The potential of this approach has been demonstrated already by exploiting the magnetic dichroism in valence band photoemission (MCD) by the excitation with $266 \mathrm{~nm}$ laser light [29]. As a certain complication the magnitude of the MCD signal depends very sensi-
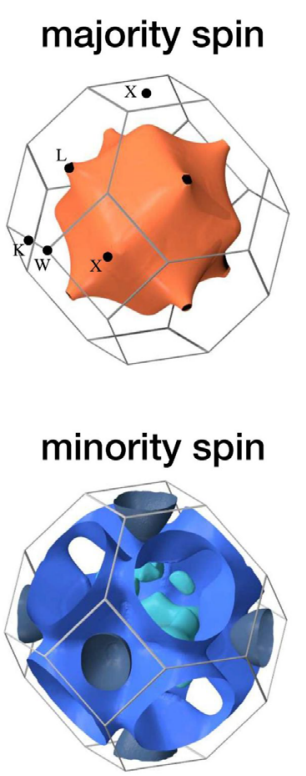
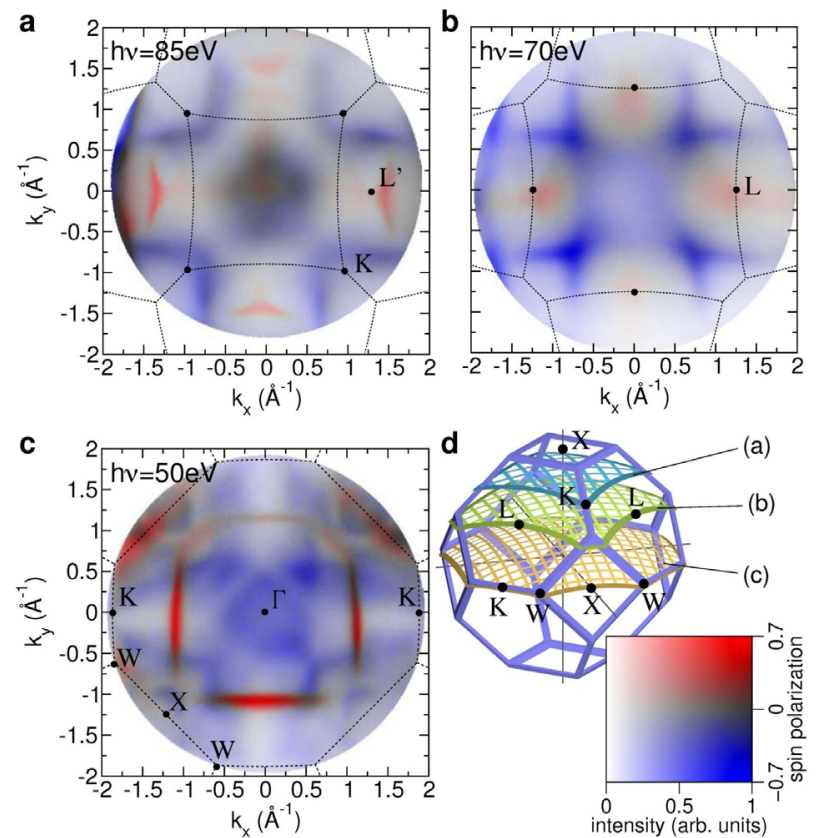

Figure 4: Spin-resolved momentum maps from $\mathrm{Co}(001)$ of a momentum microscope taken at (a) $h v=85 \mathrm{eV}$, (b) $70 \mathrm{eV}$, and (c) $50 \mathrm{eV}$ photon energies. (d) Position of the momentum maps in the bulk Brillouin zone and spin polarization color encoding. Left: Majority and minority spin Fermi surfaces of fcc cobalt. 
tively on the details of the electronic band structure, the strength of the spin-orbit coupling and may vary strongly between elements. It represents a more indirect measurement of the spin state. The photoelectron spin polarization instead yields a direct access to the spin states in the sample.

\section{B. Momentum space imaging}

Setting the immersion lens optics to image the angular distribution of photoelectrons from the sample gives direct access to the electronic states in the Fermi volume. This is demonstrated in the spin-resolved photoelectron distributions recorded from ultrathin epitaxial Cobalt films on $\mathrm{Cu}(001)$ [30]. The data reproduced in Figure 4 have been recorded with synchrotron radiation of different photon energies and map the states at the Fermi level $E_{\mathrm{F}}$. For comparison, we also show calculated Fermi surfaces for the Cobalt majority and minority spin states. The spin polarization is color-coded and can be seen to vary significantly across each photoelectron map and with the photon energies $h v=$ $85 \mathrm{eV}$ (a), $70 \mathrm{eV}$ (b), and $50 \mathrm{eV}$ (c). Each photoelectron map represents a cut through the Brillouin zone at a different position along the $\Gamma-\mathrm{X}$ direction [Figure $4(\mathrm{~d})$ ]. Because of momentum conservation in photoemission, these cuts lie on curved surfaces.

While the geometry of the Fermi surface agrees usually well with theoretical predictions, many-body interactions which are only partially captured in an independent-particle picture lead to an energy renormalization and to a finite lifetime. As a quasi-particle spends only a limited time in one state before being scattered, it follows from Heisenberg's uncertainty principle that also its energy is not sharply defined, resulting in strongly broadened states. In ferromagentic materials, these electron many-body correlations become spin dependent [31].

\section{Imaging spin-dependent correlations}

The data in Figure $4(a-c)$ only show the top slices of three-dimensional data sets which have been recorded as a function of binding energy. The high density of this data set permits access to minute details of the electronic structure and enabled a precise investigation of spin-dependent correlation effects throughout the entire Brillouin zone [30]. The development of the spin-resolved spectral function for larger binding energies is shown in Figure 5.

As a consequence of the pronounced spin-dependent electron correlations in cobalt, the well-defined contours of the majority and minority electron states observed in the Fermi surface get smeared out, particularly for the majority electron states, already a few $100 \mathrm{meV}$ below the Fermi energy. In the measured spin-resolved momentum disks this is clearly observed as the red colored majority electron background that extends over the whole Brillouin zone.

The right half of each panel in Figure 5 shows the result of theoretical photoemission calculations side-by-side with the experiment. We find a remarkably good agreement of all prominent features in the complete energy and momentum range. Particularly the onset of a strong lifetime broadening of majority electron states and the location of emission features in the Brillouin zone is well reproduced. The quantitative agreement with the experiment directly showed the importance of including a spin-dependent non-local self-energy $\Sigma^{\uparrow \downarrow}(E, \vec{k})$ to properly describe the photoemission spectra at higher binding energies.

\section{Complex spin textures}

The geometry of the spin-dependent scattering in the imaging spin filter defines a quantization axis perpendicular to the scattering plane. As a consequence, the spin detector can only determine the component of the spin polarization vector $\vec{P}=\left(P_{x}, P_{y}, P_{z}\right)$ along this quantization axis. Due to the geometry of the momentum microscope, this spin polarization component translates back to spins being oriented within the sample surface, for instance, $P_{x}$. Rotation of the sample by $90^{\circ}$ will then give access to the component $P_{y}$.

However, quantum materials such as, for example, topological insulators and Dirac heavy metals may exhibit complex spin structures in momentum space, which are caused by spin-momentum locking [32]. Figure 6 shows the spin texture on the (110) surface of the heavy metal tungsten. Spin-momentum locking here leads to highly polarized states in the Fermi surface contour, extending also to higher
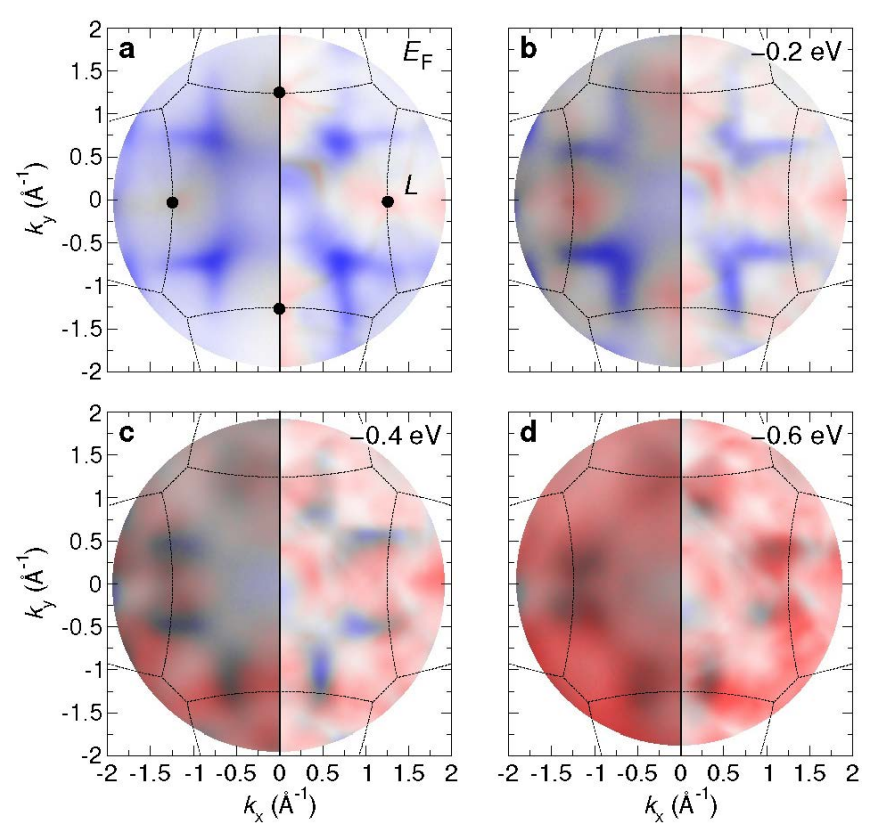

Figure 5: Spin-resolved momentum maps from $\mathrm{Co}(001)$ measured at $h v=70 \mathrm{eV}$ (left half) and calculated photoemission maps (right half). A pronounced increase of majority background intensity is observed at binding energies between $-0.2 \mathrm{eV}$ and $-0.4 \mathrm{eV}(\mathrm{b}-\mathrm{d})$, indicating the particularly short majority state lifetime few 100 meV below $E_{\mathrm{F}}(\mathrm{a})$. Position and broadening of majority and minority features are well reproduced in the calculation using a non-local self energy. 


\section{eJSSNT}

a

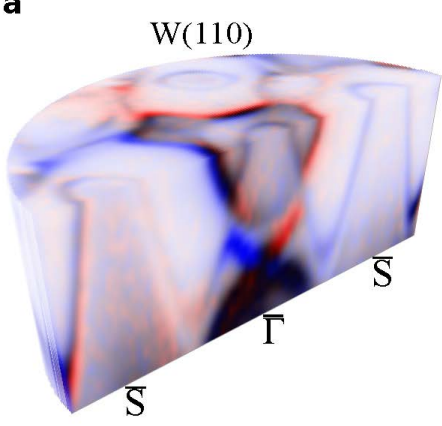

b

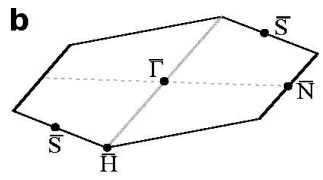

C

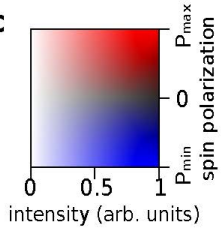

Figure 6: Three-dimensional volume map (a) of the spin-resolved electron bands at the W(110) crystal surface. The measurement shows several highly spin-polarized surface states and surface resonances in the Fermi surface of $\mathrm{W}(110)$. The section along the $\overline{\mathrm{S}}-\bar{\Gamma}-\overline{\mathrm{S}}$ direction in the surface Brillouin zone (b) reveals a Dirac like topological surface state at the $\bar{\Gamma}$ point. The intensity and spin-polarization is simultaneously displayed using a $2 \mathrm{D}$ color code (c). The full three-dimensional data set was measured at the NanoEsca beamline [23] at the Elettra synchrotron (Trieste, Italy) within only $2 \mathrm{~h}$ acquisition time.

binding energies. Resembling the metal counterpart of an topological insulator, a non-trivial Dirac cone surface state exists around the $\bar{\Gamma}$ point in the surface Brillouin zone [Figure 6(b)], located in an spin-orbit induced bulk band gap [33]. The corresponding spin polarized Dirac cone states are clearly visible in the band cross section in Figure 6(a). In addition the W(110) surface hosts a second, tilted Dirac cone state along the $\bar{\Gamma}-\overline{\mathrm{N}}$ direction [25], as well as several Rashba-type spin-orbit split states around the $\bar{\Gamma}$ and $\overline{\mathrm{S}}$ points.

Capturing such complex spin textures in detail requires a determination of the full spin polarization vector $\vec{P}\left(E_{\mathrm{B}}, \vec{k}\right)$. This can be achieved by installing a spin-rotator in front of the spin filter. Such spin-rotators have already been successfully employed in spin-polarized low energy electron microscopy (SPLEEM) [13]. However, in a SPLEEM the spin rotator sits in the illumination column, whereas in the momentum microscope it has to be placed in the imaging column, i.e., while rotating the spin the device must preserve the image.

\section{E. High-energy momentum microscopy}

In our discussion of momentum microscopy up to now we have implicitly assumed the typical ARPES regime of photon energies, i.e., of the order of up to $150 \mathrm{eV}$. This type of experiment is very surface-sensitive, due to the small inelastic mean free path of the photoelectrons. In order to increase the bulk sensitivity of the photoemission technique, one has to go to higher photon energies in the (hard) X-ray regime, i.e., of the order of several $\mathrm{keV}$. This has established the field of hard X-ray photoelectron spectroscopy (HAXPES) [34]. The large information depth of the hard X-ray approach has also been successfully demonstrated in PEEM [35]. In an effort to enabling HAXPES studies with lateral resolution, we have established the first energy-filtered photoelectron microscope at the synchrotron facility PETRA-III (Hamburg) [36, 37]. The electron-optical layout of the instrument is similar to the system installed at Elettra, however, with the optics adapted to higher kinetic energies. The instrument is mainly used for material science studies in information and energy research and a recent example is given in Figure 7. It represents a spectromicroscopic study of the resistive switching in $\mathrm{ZrO}_{x}$ [38]. The PEEM image [Figure 7(a)] has been recorded with $\mathrm{Zr} 3 \mathrm{~d}$ photoemission at a photon energy of $h v=3 \mathrm{keV}$ and shows a top view of the junction including the contact electrodes. The layer stack in the junction area (red square) contains as active resistive element a bilayer of $\mathrm{ZrO}_{x} / \mathrm{Ta}$, with the electrical contacts provided by $\mathrm{Pt}$ electrodes. The $\mathrm{Zr} 3 \mathrm{~d}$ core level HAXPES spectra acquired in a reference region next to the junction (green square) indicated a predominant $\mathrm{Zr}^{4+}$ valence state compatible with $\mathrm{ZrO}_{2}$. The resistive switching leads to the buildup of a $\mathrm{Zr}^{3+}$ component in the junction area (red square), indicating the formation of a suboxide. A similar behavior is observed in the Ta 4f HAXPES spectra from the junction area [Figure 7(b)]. A principal component analysis of the data yields several spectral contributions, resulting from tantalum oxides with approximate Ta valence states $\mathrm{Ta}^{5+}\left(\mathrm{Ta}_{2} \mathrm{O}_{5}\right), \mathrm{Ta}^{4+}\left(\mathrm{TaO}_{2-x}\right)$, and $\mathrm{Ta}^{2+}\left(\mathrm{TaO}_{1-x}\right)$. This indicates that Tantalum strongly participates in the resistive switching and the associated redox and oxygen exchange processes.

The spectra recorded for the HRS [Figure 7(c)] and LRS state [Figure 7(d)] exhibit subtle differences, which are related to the local redox reaction during the switching processes. A careful analysis reveals that the changes are taking place mainly between the $\mathrm{TaO}_{2-x}$ and $\mathrm{TaO}_{1-x}$ suboxides. This example illustrates the power but also limits of the current
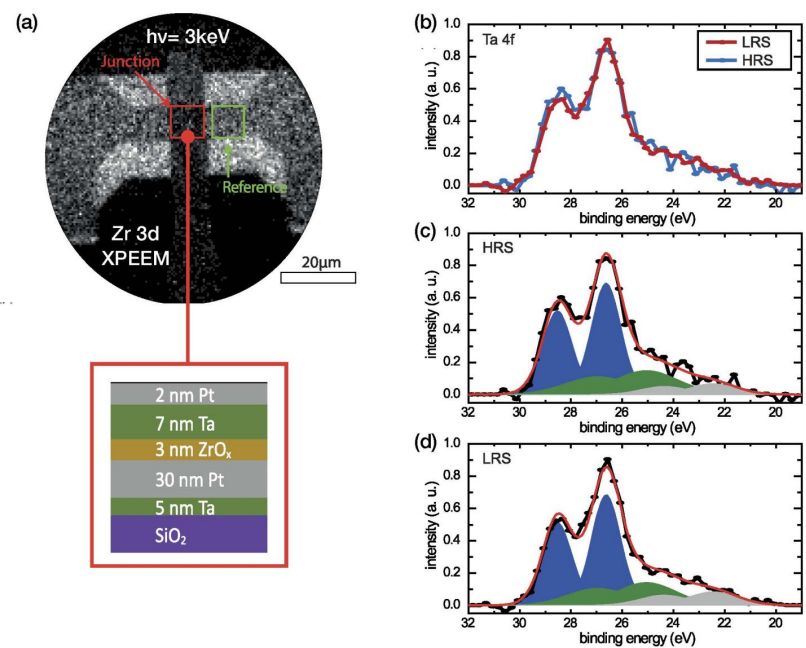

Figure 7: HAXPES spectromicroscopy from $\mathrm{ZrO}_{x} / \mathrm{TaO}_{x}$ switching contacts. (a) PEEM image recorded with the $\mathrm{Zr} 3 \mathrm{~d}$ electrons at $h v$ $=3 \mathrm{keV}$. Inset: cross section of the layer stack in the junction area (red square). (b) Ta $4 \mathrm{f}$ spectra in the HRS and LRS states, respectively. (c) Spectral contributions of the various suboxides in the junction area extracted from a principal component analysis for the HRS configuration. (d) Same for LRS. Adapted from Ref. 38. 
HAXPES spectromicroscopy.

Using HAXPES to study band dispersions is complicated by the fact that phonon contributions and the finite photon momentum have to be taken into account [39]. However, if this is done properly, it is indeed possible to obtain momentum-resolved maps of the bulk electronic states [40]. Therefore, despite the low photoexcitation cross sections at high photon energies also momentum microscopy in the HAXPES regime should be feasible. This was demonstrated very recently using a time-of-flight spectrometer [41].

\section{F. Imaging ultrafast dynamics}

Going beyond the imaging of static states and being able to address dynamic processes is one of the grand challenges in electron microscopy at large. Time-resolved imaging of magnetization dynamics was successfully performed in XPEEM addressing the 100-ps time scale [42]. A momentum microscope images the electronic structure directly and should therefore be able to address electronic time scales of the order of $100 \mathrm{fs}$ and less. This calls for pump-probe experiments with ultrashort pulsed laser sources. A first experiment with a ToF-based momentum microscope operated at the free electron laser source FLASH (Hamburg) without spin-resolution has already demonstrated the feasibility of this approach [43]. Time-and spin-resolved photoemission experiments of the ultrafast demagnetization in ferromagnets have recently revealed the spin dynamics at selected points in $k$-space [44]. These experiments used a pump-probe scheme with a $h v=1.6 \mathrm{eV}$ pump pulse and a high-harmonic generation stage to generate 50 fs pulses with $h v=22.5 \mathrm{eV}$ for the ARPES analysis. The question arises whether the response observed is representative for the dynamics in the entire Brillouin zone. In fact, theoretical work indicates that the dynamic response of the electron and spin system may vary strongly throughout the Brillouin zone [45]. In particular, the calculation predicts several "hot spots", where the dynamic spin changes are much higher than in the rest of the Brillouin zone. This is the kind of situation where spin- and time-resolved momentum microscopy can unfold its full potential and we are currently realizing a set-up with a high-repetition high-harmonic source in our lab.

\section{CONCLUSIONS}

During recent years spin-resolved momentum microscopy has matured into a powerful tool for the detailed study of electronic structures. The capability to efficiently collect high-density data sets throughout the entire Brillouin zone enables unparalleled access to minute details of the electronic states and their spin character. An "All-in-One" experiment as outlined in Figure 8 is already within reach, with two more capabilities to be added; (i) a determination of the full spin polarization vector $\vec{P}$, and (ii) a time-resolution covering the typical time scales of the electron and spin subsystems. Respective development activities in our institute are under way.

\section{Acknowledgments}

We are indeped to Marek Wilhelm, Tristan Heider, Bharti Parashar, Kenta Hagiwara, Xin Tan, Matteo Jugovac, Giovanni Zamborlini, and Vitaliy Feyer for their collaboration. Funding by BMBF (grant nos. 05K16PGB and 05K19PGA) is gratefully acknowledged.

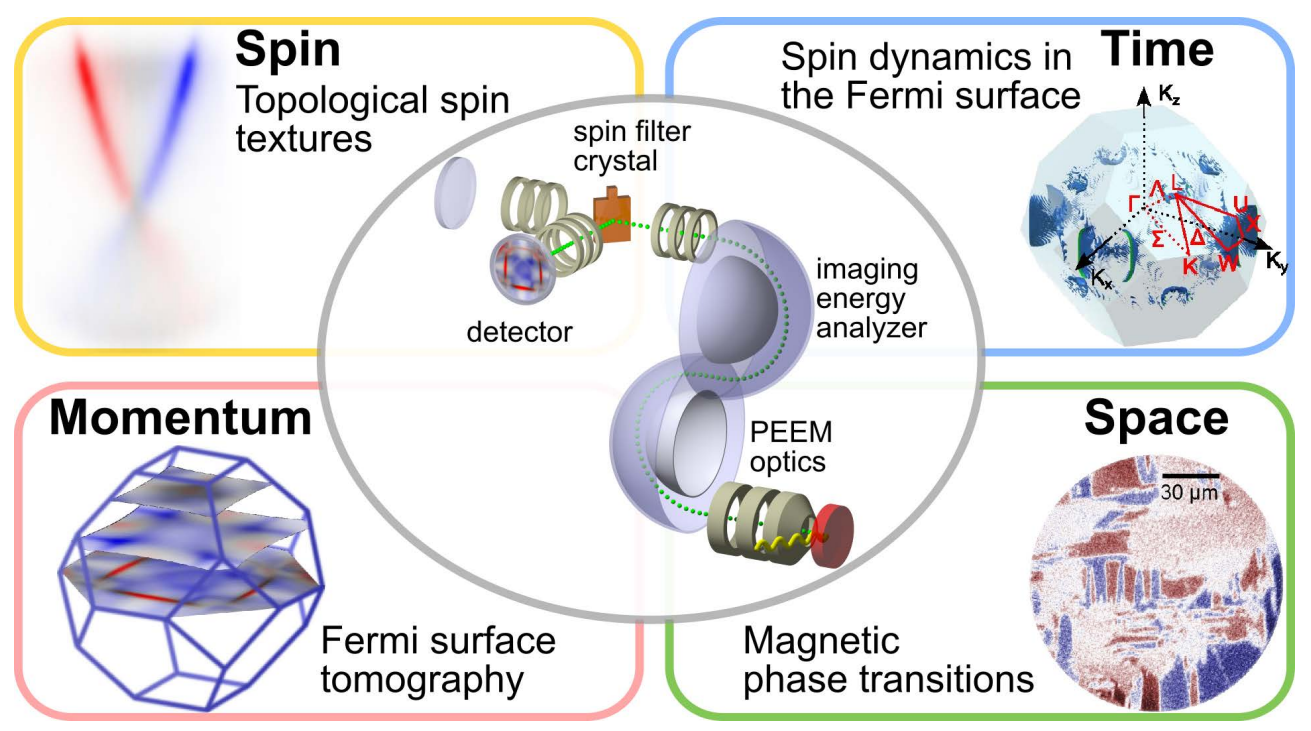

Figure 8: The "All-in-One" photoemission experiment combines information on the surface geometry in real space, momentum resolved maps of the electron states and complete Fermi surfaces, complex spin textures as well as time dependent dynamics [11, 27, 30, 45] in the femtosecond regime. The momentum microscope [21] bridges all these dimensions in a single experiment, with the ultrafast timescale becoming available with the advent of ultrafast laser driven light sources and free-electron lasers. 


\section{ALC '19 Conference}

Note

This paper was presented at the 12th International Symposium on Atomic Level Characterizations for New Materials and Devices '19 (ALC '19), in conjunction with the 22nd International Conference on Secondary Ion Mass Spectrometry (SIMS-22), Miyako Messe, Kyoto, Japan, 20-25 October, 2019.

\section{References}

[1] B. Keimer and J. E. Moore, Nat. Phys. 13, 1045 (2017).

[2] N. Gedik and I. Vishik, Nat. Phys. 13, 1029 (2017).

[3] T. Shimojima, K. Okazaki, and S. Shin, J. Phys. Soc. Jpn. 84, 072001 (2015).

[4] M. Amati, A. Barinov, V. Feyer, L. Gregoratti, M. Al-Hada, A. Locatelli, T. O. Mentes, H. Sezen, C. M. Schneider, and M. Kiskinova, J. Electron Spectros. Relat. Phenomena 224, 59 (2018).

[5] K. Nakayama, S. Souma, C. X. Trang, D. Takane, C. Chen, J. Avila, T. Takahashi, S. Sasaki, K. Segawa, M. C. Asensio, Y. Ando, and T. Sato, Nano Lett. 19, 3737 (2019).

[6] J. Kirschner, in: Polarized Electrons in Surface Physics, edited by R. Feder (World Scientific, Singapore, 1986).

[7] A. Winkelmann, D. Hartung, H. Engelhard, C.-T. Chiang, and J. Kirschner, Rev. Sci. Instrum. 79, 83303 (2008).

[8] L. Plucinski, A. Oelsner, F. Matthes, and C. M. Schneider, J. Electron Spectros. Relat. Phenomena 181, 215 (2010).

[9] M. Escher, N. B. Weber, M. Merkel, L. Plucinski, and C. M. Schneider, e-J. Surf. Sci. Nanotechnol. 9, 340 (2011).

[10] T. Heider, E. Mlynczak, L. Plucinski, K. Grasza, P. Skupinski, and A. Wolos (unpublished).

[11] H. L. Meyerheim and C. Tusche, Phys. Status Solidi RRL 12, 1800078 (2018).

[12] E. Brüche, Z. Phys. 86, 448 (1933).

[13] E. Bauer, Surface Microscopy with Low Energy Electrons (Springer, New York, 2014).

[14] M. Wilhelm, M. Giesen, M. Moors, Ch. Bäumer, M. Hussein Hamed, J. Hackl, L. Cao, H. Zhang, T. Duchon, M. Glöß, O. Petracic, C. Wiemann, S. Cramm, S. Nemsak, R. Dittmann, C. M. Schneider, and M. Müller (unpublished).

[15] D. Ielmini and R. Waser (Eds.), Resistive Switching: From Fundamentals of Nanoionic Redox Processes to Memristive Device Applications (Wiley-VCH Verlag, 2016).

[16] B. Krömker, M. Escher, D. Funnemann, D. Hartung, H. Engelhard, and J. Kirschner, Rev. Sci. Instrum. 79, 053702 (2008).

[17] A. Oelsner, O. Schmidt, M. Schicketanz, M. Klais, G. Schönhense, V. Mergel, O. Jagutzki, and H. Schmidt-Böcking, Rev. Sci. Instrum. 72, 3968 (2001).

[18] K. Medjanik, O. Fedchenko, S. Chernov, D. Kutnyakhov, M. Ellguth, A. Oelsner, B. Schönhense, T. R. F. Peixoto, P. Lutz, C. H. Min, F. Reinert, S. Däster, Y. Acremann, J. Viefhaus, W. Wurth, H. J. Elmers, and G. Schönhense, Nat. Mater. 16, 615 (2017).

[19] M. Kolbe, P. Lushchyk, B. Petereit, H. J. Elmers, G. Schönhense, A. Oelsner, C. Tusche, and J. Kirschner, Phys. Rev. Lett. 107, 207601 (2011).

[20] C. Tusche, M. Ellguth, A. A. Ünal, C.-T. Chiang, A. Winkelmann, A. Krasyuk, M. Hahn, G. Schönhense, and J. Kirschner, Appl. Phys. Lett. 99, 032505 (2011).

[21] C. Tusche, A. Krasyuk, and J. Kirschner, Ultramicroscopy 159, 520 (2015).

[22] C. Tusche, Y.-J. Chen, C. M. Schneider, and J. Kirschner, U1tramicroscopy 206, 112815 (2019).
[23] C. Wiemann, M. Patt, I. P. Krug, N. B. Weber, M. Escher, M. Merkel, and C. M. Schneider, e-J. Surf. Sci. Nanotechnol. 9, 395 (2011).

[24] M. Büscher, R. Adam, C. Tusche, A. Hützen, C. Wiemann, Y.-J. Chen, and C. M. Schneider, J. Large-Scale Res. Facil. 6, A138 (2020).

[25] D. Kutnyakhov, S. Chernov, K. Medjanik, R. Wallauer, C. Tusche, M. Ellguth, S. A. Nepijko, M. Krivenkov, J. Braun, S. Borek, J. Minár, H. Ebert, H. J. Elmers, and G. Schönhense, Sci. Rep. 6, 29394 (2016).

[26] X. M. Cheng and D. J. Keavney, Rep. Prog. Phys. 75, 026501 (2012).

[27] J. de la Figuera and C. Tusche, Appl. Surf. Sci. 391, 66 (2017). [28] A. Locatelli, T. O. Menteş, M. Á. Niño, and E. Bauer, Ultramicroscopy 111, 1447 (2011).

[29] T. Taniuchi, Y. Kotani, and S. Shin, Rev. Sci. Instrum. 86, 023701 (2015).

[30] C. Tusche, M. Ellguth, V. Feyer, A. Krasyuk, C. Wiemann, J. Henk, C. M. Schneider, and J. Kirschner, Nature Commun. 9, 3727 (2018).

[31] J. Sánchez-Barriga, J. Fink, V. Boni, I. Di Marco, J. Braun, J. Minár, A. Varykhalov, O. Rader, V. Bellini, F. Manghi, H. Ebert, M.

I. Katsnelson, A. I. Lichtenstein, O. Eriksson, W. Eberhardt, and H.

A. Dürr, Phys. Rev. Lett. 103, 267203 (2009).

[32] M. S. Bahramy, P. D. C. King, A. de la Torre, J. Chang, M. Shi, L. Patthey, G. Balakrishnan, P. Hofmann, R. Arita, N. Nagaosa, and F. Baumberger, Nat. Commun. 3, 1159 (2012).

[33] K. Miyamoto, A. Kimura, K. Kuroda, T. Okuda, K. Shimada, H. Namatame, M. Taniguchi, and M. Donath, Phys. Rev. Lett. 108, 066808 (2012).

[34] J. C. Woicik (Ed.), Hard X-ray Photoelectron Spectroscopy (HAXPES) (Springer International Publishing, Switzerland, 2016).

[35] T. Wakita, T. Taniuchi, K. Ono, M. Suzuki, N. Kawamura, M. Takagaki, H. Miyagawa, F. Guo, T. Nakamura, T. Muro, H. Akinaga, T. Yokoya, M. Oshima, and K. Kobayashi, Jpn. J. Appl. Phys. 45, 1886 (2006).

[36] M. Patt, C. Wiemann, N. Weber, M. Escher, A. Gloskovskii, W. Drube, M. Merkel, and C. M. Schneider, Rev. Sci. Instrum. 85, 113704 (2014).

[37] C. Schlueter, A. Gloskovskii, K. Ederer, I. Schostak, S. Piec, I. Sarkar, Y. Matveyev, P. Lömker, M. Sing, R. Claessen, C. Wiemann, C. M. Schneider, K. Medjanik, G. Schönhense, P. Amann, A. Nilsson, and W. Drube, AIP Conf. Proc. 2054, 040010 (2019).

[38] A. Kindsmüller, C. Schmitz, C. Wiemann, K. Skaja, D. J. Wouters, R. Waser, C. M. Schneider, and R. Dittmann, APL Mater. 6, 046106 (2018).

[39] L. Plucinski, J. Minár, B. C. Sell, J. Braun, H. Ebert, C. M. Schneider, and C. S. Fadley, Phys. Rev. B 78, 035108 (2008).

[40] A. X. Gray, C. Papp, S. Ueda, B. Balke, Y. Yamashita, L. Plucinski, J. Minár, J. Braun, E. R. Ylvisaker, C. M. Schneider, W. E. Pickett, H. Ebert, K. Kobayashi, and C. S. Fadley, Nat. Mater. 10, 759 (2011).

[41] S. Babenkov, K. Medjanik, D. Vasilyev, S. Chernov, C. Schlueter, A. Gloskovskii, Y. Matveyev, W. Drube, B. Schönhense, K. Rossnagel, H.-J. Elmers, and G. Schönhense, Comm. Phys. 2, 107 (2019).

[42] G. Schönhense, H. J. Elmers, S. A. Nepijko, and C. M. Schneider, Adv. Imag. Electron Phys. 142, 159 (2006).

[43] D. Kutnyakhov, R. P. Xian, M. Dendzik, M. Heber, F. Pressacco, S. Y. Agustsson, L. Wenthaus, H. Meyer, S. Gieschen, G. Mercurio, A. Benz, K. Bühlman, S. Däster, R. Gort, D. Curcio, K. Volckaert, M. Bianchi, Ch. Sanders, J. A. Miwa, S. Ulstrup, A. 
Oelsner, C. Tusche, Y.-J. Chen, D. Vasilyev, K. Medjanik, G. Brenner, S. Dziarzhytski, H. Redlin, B. Manschwetus, S. Dong, J. Hauer, L. Rettig, F. Diekmann, K. Rossnagel, J. Demsar, H.-J. Elmers, Ph. Hofmann, R. Ernstorfer, G. Shönhense, Y. Acremann, and W. Wurth, Rev. Sci. Instrum. 91, 013109 (2020).

[44] S. Eich, M. Plötzing, M. Rollinger, S. Emmerich, R. Adam, C. Chen, H. C. Kapteyn, M. M. Murnane, L. Plucinski, D. Steil, B. Stadtmüller, M. Cinchetti, M. Aeschlimann, C. M. Schneider, and S. Mathias, Sci. Adv. 3, e1602094 (2017).

[45] M. S. Si, J. Y. Li, D. Z. Yang, D. S. Xue, and G. P. Zhang, Sci. Rep. 4, 5010 (2015).

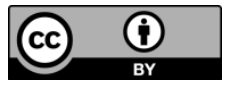

All articles published on e-J. Surf. Sci. Nanotechnol. are licensed under the Creative Commons Attribution 4.0 International (CC BY 4.0). You are free to copy and redistribute articles in any medium or format and also free to remix, transform, and build upon articles for any purpose (including a commercial use) as long as you give appropriate credit to the original source and provide a link to the Creative Commons (CC) license. If you modify the material, you must indicate changes in a proper way.

Published by The Japan Society of Vacuum and Surface Science 